\title{
GBA mutations and Parkinson's disease in Brazil
}

\author{
Mutações do GBA e doença de Parkinson no Brasil \\ Carlos R. M. Rieder ${ }^{1,2}$
}

${ }^{1}$ Universidade Federal Ciências da

Saúde de Porto Alegre, Santa Casa de Misericórdia de Porto Alegre,

Porto Alegre RS, Brasil;

${ }^{2}$ Hospital de Clínicas de Porto

Alegre, Porto Alegre RS, Brasil.

Correspondence:

Carlos R. M. Rieder; Rua Padre

Chagas, 147 / conj 602; 90570-080

Porto Alegre RS, Brasil;

E-mail: carlosrieder@gmail.com

https://orcid.org/0000-00032950-7211

Conflict of interest:

There is no conflict of interest to declare.

Received 18 January 2019; Accepted 25 January 2019.

\section{(c) BY}

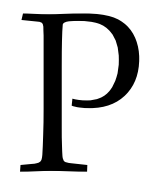

arkinson's disease (PD) is a complex neurodegenerative disease. Remarkable advances in the understanding of genetic risk factors underlying the pathogenesis of PD have occurred in the last decades when more than 20 genes have been identified as causes of PD. One of the most important discoveries was that mutations in the glucocerebrosidase (GBA) gene, coding for the lysosomal enzyme glucocerebrosidase, have a substantial role in PD.

Homozygous GBA mutations are known as the cause of Gaucher disease, which is the most common lysosomal storage disease worldwide, and has a particularly high prevalence among Ashkenazi Jews. However, heterozygous GBA mutations have been found, even in early studies, to be significantly more frequent in PD patients compared with controls. This was observed in screenings done in Ashkenazi Jews ${ }^{1}$ and in a large-scale screening of European PD patients ${ }^{2}$. Therefore, after many clinical, neuropathologic, and genetic studies, it has been very well established that heterozygous mutations in this gene are also important risk factors for PD. Furthermore, mutations of the GBA gene are considered, nowadays, the most significant risk factor for $\mathrm{PD}$, and are the most common genetic mutation so far identified in this disease.

The molecular mechanisms by which $G B A$ mutations result in this increased risk has been an important research focus. One hypothesis is that there is a reduction in enzymatic activity leading to an increase in the levels of glucosylceramide in specific regions of the brain. A significant reduction in substantia nigra and striatum glucocerebrosidase activity was identified in the brain of PD patients. This was related to the abnormal accumulation of $\alpha$-synuclein, alterations in lysosomal autophagy and chaperone-mediated autophagy and lipid metabolism. Consequently, the GBA-lysosomal-synuclein axis has been the focus of experimental therapeutics for the development of novel drugs to modulate glucocerebrosidase activity.

The highest frequencies of mutations of the GBA gene have been found in PD patients of Ashkenazi Jewish ancestry in comparison with control groups. The frequencies are much lower in PD patients from non-Jewish populations. However, the penetrance of GBA-related $\mathrm{PD}$ is variable in both populations, Jewish and non-Jewish. This penetrance variability is possibly the main unanswered point in the understanding of PD and a major obstacle to development of neuroprotective treatment before the onset of motor symptoms. A family history of PD is the single greatest risk factor for developing PD, although only a small minority of patients develop PD in a Mendelian manner. Variable penetrance may be related to different environmental factors and/or epigenetic factors. Therefore, is very important to study the frequency of PD associated with GBA mutations in different environments and in different genetic backgrounds.

Several variants in the $G B A$ gene have been identified as risk factors for PD. There is a difference in the prevalence of this mutation in various ethnic groups and countries. The p.L4444P and p.N370S are the most-widely analyzed in studies of the association between mutations of the GBA gene and PD.

In this issue of Arquivos de Neuropsiquiatria, Amaral and colleagues present a casecontrol study of the occurrence of the two most common GBA mutations (c.1226A > G; p.N370S and c.1448T > C; p.L444P) in patients from northern Brazil ${ }^{3}$. The authors examined 
a group of $81 \mathrm{PD}$ patients and 81 control individuals. In the patients group they found that $3.7 \%$ were heterozygous for the $G B A$ p.N370S mutation, with the same frequency (3.7\%) for $G B A$ p.L444P. Neither mutation was detected in the control group. Their results from northern Brazil are similar to other studies from different Brazilian regions ${ }^{4.5,6}$.

In the Amaral et al. ${ }^{3}$ study, all six PD patients with one of the mutations presented clinically as a typical PD phenotype. The authors found that patients with p.L444P mutation showed a tendency to present with earlier disease onset. This agrees with other studies that found that $G B A$ heterozygous patients have an earlier age at onset compared with noncarriers. However, some studies have not found this difference. The authors also reported that no cognitive dysfunction or psychiatric disturbances were found. Some studies have suggested that PD patients carrying GBA variants have a faster progression to dementia, with carriers of deleterious mutations displaying a more rapid disease progression than carriers of GBA polymorphisms. The reasons for these clinical differences between studies are not clear.

The authors point out that clinical differences may be accounted for by the fact that the modifier genes that contribute to development of the phenotype of the disease may also vary systematically in different populations. This is, by itself, one important reason to study GBA risk factors in different populations. Thus, due to the wide diversity between different Brazilian regions, research across the country is necessary in order to properly characterize GBA mutations in our population.

The Amaral et al. ${ }^{3}$ study further reinforces the association of mutations of the $G B A$ gene as a factor of genetic susceptibility for the development of PD in the Brazilian population.

\section{References}

1. Sidransky E, Nalls MA, Aasly JO, Aharon-Peretz J, Annesi G, Barbosa ER, et al. Multicenter analysis of glucocerebrosidase mutations in Parkinson's disease. N Engl J Med. 2009 Oct;361(17):1651-61. https://doi.org/10.1056/NEJMoa0901281

2. Lesage S, Anheim M, Condroyer C, Pollak P, Durif F, Dupuits C, et al. Large-scale screening of the Gaucher's disease-related glucocerebrosidase gene in Europeans with Parkinson's disease. Hum Mol Genet. 2011 Jan;20(1):202-10. https://doi.org/10.1093/hmg/ddq454

3. Amaral CE. Amaral, Lopes PF, Ferreira JC et al. GBA mutations p.N370S and p.L444P are associated with Parkinson disease in patients from Northern Brazil. Arq Neuropsiquiatr. 2019;77(2):73-9. https://doi.org/10.1590/0004-282X20190006
4. Spitz M, Rozenberg R, Pereira LV, Reis Barbosa E. Association between Parkinson's disease and glucocerebrosidase mutations in Brazil. Parkinsonism Relat Disord. 2008;14(1):58-62. https://doi.org/10.1016/j.parkreldis.2007.06.010

5. Socal MP, Bock H, Michelin-Tirelli K, Hilbig A, SaraivaPereira ML, Rieder CR, et al. Parkinson's disease and the heterozygous state for glucocerebrosidase mutations among Brazilians. Parkinsonism Relat Disord. 2009 Jan;15(1):76-8. https://doi.org/10.1016/j.parkreldis.2008.01.019

6. Guimarães BC, Pereira AC, Rodrigues FC, dos Santos AV, Campos Junior M, Santos JM, et al. Glucocerebrosidase N370S and L444P mutations as risk factors for Parkinson's disease in Brazilian patients. Parkinsonism Relat Disord. 2012 Jun;18(5):688-9. https://doi.org/10.1016/j.parkreldis.2011.11.028 